\title{
Ultrawide Bandwidth Signals as Shot Noise: A Unifying Approach
}

\author{
Andrea Ridolfi, Student Member, IEEE, and Moe Z. Win, Fellow, IEEE
}

\begin{abstract}
We present a shot noise based model for a large family of ultrawide bandwidth (UWB) signals. These include time-hopping and direct-sequence signaling with pulse position, interval, and amplitude modulations. Each specific signal is constructed by adding features to a basic model in a modular, simple, and tractable way. Our work unifies the contributions scattered in the literature and provides a general approach that allows various extensions of previous works as well as new results. The exact power spectrum is then evaluated using shot noise spectral theory, which provides a simpler, systematic, and rigorous approach to the spectra evaluation of complicated UWB signals. The strength of our methodology is that different features of the signal model contribute clearly and separately to the resulting spectral expressions.
\end{abstract}

Index Terms-Point processes, power spectrum, pulse modulated signals, shot noise, ultrawide bandwidth.

\section{INTRODUCTION}

$\mathbf{T}$ HE family of pulse modulation techniques has gathered increasing attention since the introduction of ultrawide bandwidth (UWB) impulse radio. UWB signals are transmitted with low spectral content while maintaining the average power level required for reliable communications. As a consequence, their spectral characteristics play a key role in the design of UWB systems.

Among several contributions to spectral evaluation, we mention the computation of the power spectrum of a time-hopping, pulse position modulated signal [1]-[3], and that of the spectral density of the family of pulse interval modulated signals [4]. Such computations concern specific signal models and are performed using the classical wide-sense stationary (WSS) approach, i.e., as the Fourier transform of the correlation function. Although this is a common approach, the resulting calculations are complex and the introduction of additional random features, such as clock jitter, pulse losses or pulse distortion, requires a new computation.

Here, we show that a large family of UWB signals can be aptly modeled as a shot noise with random excitation, i.e., a filtered point process with a random filtering function. Shot noise processes have received much attention in the applied literature

Manuscript received March 1, 2005; revised October 15, 2005. This research was supported in part by the Swiss National Science Foundation under Grant 21-65187.01, in part by the Office of Naval Research Young Investigator Award N00014-03-1-0489, in part by the National Science Foundation under Grant ANI-0335256, in part by the Charles Stark Draper Endowment, and in part by the Institute of Advanced Study Natural Science and Technology Fellowship.

A. Ridolfi is with the Laboratory of AudioVisual Communications, École Polytechnique Fédérale de Lausanne, CH-1015 Lausanne, Switzerland (e-mail: andrea.ridolfi@epfl.ch).

M. Z. Win is with the Laboratory for Information and Decision Systems, Massachusetts Institute of Technology, Cambridge, MA 02139 USA (e-mail: moewin@mit.edu).

Digital Object Identifier 10.1109/JSAC.2005.863881 (see [5] and the references therein). They have been widely used in queuing and teletraffic theory [6] and in pulse coded optical transmission systems [7], [8].

There are two considerable advantages in modeling UWB signals as shot noise processes. First, the model is modular, simple, and tractable. Thus, one can construct different UWB signals in a unifying way by adding features to a basic model systematically, and can easily take into account random quantities, such as jitter, losses, or pulse distortions, affecting the signals. Second, spectra are obtained in a systematic and rigorous manner from a single general formula. This formula simplifies proofs of existing results and provides spectrum expressions where various features of the model appear separately and explicitly. These advantages have a tremendous impact on the design and analysis of UWB signals.

UWB transmission systems employ time-hopping or directsequence signals to achieve multiple-access with pulse position or pulse amplitude modulation for data transmission (see for instance [1], [9]). Here, we focus on the following schemes: pulse amplitude modulation (PAM), pulse position modulation (PPM), pulse interval modulation (PIM), time-hopping (TH) signals, and direct-sequence (DS) signals. The model allows us to also treat highly complicated signals such as pulse-modulated signals over multipath fading channels [10], [11].

The outline of the paper is as follows. Section II recalls the definition of a shot noise process and gives the general expression of its spectrum. In Sections III and IV, we introduce, respectively, UWB pulse-modulated signals and UWB multiple-access pulse-modulated signals, and we describe how these can be expressed using a single shot noise model. For each signal, we provide the corresponding spectrum as a particular case of the shot noise spectrum. Section V presents some numerical evaluations of spectra.

\section{SHOT NoISE With RANDOM EXCITATION}

A shot noise with random excitation is a stochastic process of the form

$$
X(t)=\sum_{n \in \mathbb{Z}} h\left(t-T_{n}, Z_{n}\right)
$$

where $\left\{T_{n}\right\}_{n \in \mathbb{Z}}$ is a general sequence of random times, not assumed to be Poisson, and $\left\{Z_{n}\right\}_{n \in \mathbb{Z}}$ is a sequence of independent and identically distributed (i.i.d.) random variables, associated to the random times but independent of them. In mathematical parlance, we refer to the random times as a point process, i.e., a random collection $N=\left\{T_{n}, n \in \mathbb{Z}\right\} .^{1}$ Then, the couples

\footnotetext{
${ }^{1}$ In the following, we shall use the subscript $N$ to indicate functions related to the random times $\left\{T_{n}\right\}_{n \in \mathbb{Z}}$.
} 
$\left\{\left(T_{n}, Z_{n}\right), n \in \mathbb{Z}\right\}$ form a marked point process [12]. The function $h(t, Z)$ can be interpreted as an impulse response function that depends on a random parameter $Z .^{2}$ In the deterministic case $h(t, Z)=h(t)$, and $X(t)$ can be symbolically obtained as the convolution of the impulse response $h$ with a sequence of Dirac pseudofunctions centered at random times $\left\{T_{n}\right\}_{n \in \mathbb{Z}}$, i.e.,

$$
X(t)=h * \sum_{n \in \mathbb{Z}} \delta\left(\cdot-T_{n}\right)(t) .
$$

In the case of UWB signals, the sequence of random times $\left\{T_{n}\right\}_{n \in \mathbb{Z}}$ determines the temporal structure of the signal and the random function $h(t, Z)$ characterizes the shape of the pulses and its random modifications (amplitude, displacement, and distortion).

Random i.i.d. modulation of the pulse amplitude can be described by the random parameter $Z$. In the more general correlated case, pulse amplitudes are modeled as a WSS time series $\left\{A_{n}\right\}_{n \in \mathbb{Z}}$ (independent of the random times) and the (modulated) shot noise with random excitation can be written as

$$
X(t)=\sum_{n \in \mathbb{Z}} A_{n} h\left(t-T_{n}, Z_{n}\right)
$$

which has (1) as a special case. We refer to $\mathbb{E}\{A\}=\mathbb{E}\left\{A_{n}\right\}$ as the mean, $\mathbb{E}\left\{A^{2}\right\}=\mathbb{E}\left\{A_{n}^{2}\right\}$ as the second order moment, and $R_{A}(k)=\mathbb{E}\left\{A_{n+k} A_{n}^{*}\right\}$ as the correlation of the WSS time series.

The power spectral density of a shot noise with random excitation is given by the following result [10].

Theorem II.1: Let $X(t)$ be the shot noise with random excitation of (1). Denote with $\widehat{h}(\nu, Z)$ the Fourier transform of $h(t, Z)$ (with respect to $t$ ) and with $\mathcal{S}_{N}(\nu)$ the power spectral density of the sequence of random times $\left\{T_{n}\right\}_{n \in \mathbb{Z}}$. Call $\lambda$ the intensity of the point process, i.e., the average number of random points, or times, per unit of time. Then

$$
\mathcal{S}_{X}(\nu)=|\mathbb{E}\{\widehat{h}(\nu, Z)\}|^{2} \mathcal{S}_{N}(\nu)+\lambda \operatorname{Var}\{\widehat{h}(\nu, Z)\} .
$$

Proof: The proof is sketched in Appendix. A more mathematically detailed proof can be found in [10].

Let now $X(t)$ be the shot noise with random excitation where the amplitudes of the shots are modulated by the time series $\left\{A_{n}\right\}_{n \in \mathbb{Z}}$, as in (2). Assume that the sequence of random times $\left\{T_{n}\right\}_{n \in \mathbb{Z}}$ forms a renewal process and denote with $\phi_{S}^{k}(u)$ the characteristic function ${ }^{3}$ of the i.i.d. sequence of inter-arrival times $S_{n}=T_{n+1}-T_{n}, n \neq 0$ (i.i.d. inter-arrival times is a property of renewal processes [13]). The power spectrum of the modulated shot noise with random excitation (2) is given by

$\mathcal{S}_{X}(\nu)=|\mathbb{E}\{\widehat{h}(\nu, Z)\}|^{2} \mathcal{S}_{N_{A}}(\nu)+\lambda \mathbb{E}\left\{|A|^{2}\right\} \operatorname{Var}\{\widehat{h}(\nu, Z)\}$

where

$$
\begin{aligned}
\mathcal{S}_{N_{A}}(\nu)= & \lambda 2 \operatorname{Re}\left\{\sum_{k \geq 0} \phi_{S}^{k}(\nu) R_{A}(k)\right\} \\
& -\lambda R_{A}(0)-\lambda^{2} \mathbb{E}\{A\}^{2} \delta(\nu) .
\end{aligned}
$$

${ }^{2} Z$ is a random variable distributed as the common distribution of the sequence $\left\{Z_{n}\right\}_{n \in \mathbb{Z}}$.

${ }^{3}$ The characteristic function is evaluated at $2 \pi u$, i.e., $\phi_{S}^{k}(u)=\mathbb{E}\left\{e^{i 2 \pi u S}\right\}$.
We recall that the power spectral density of a renewal process is given by [12]

$$
\mathcal{S}_{N}(\nu)=\lambda\left[2 \operatorname{Re}\left\{\sum_{k \geq 0} \phi_{S}^{k}(\nu)\right\}-1\right]-\lambda^{2} \delta(\nu) .
$$

In particular, a regularly $T$-spaced grid is a special case of renewal process, with

$$
\lambda=\frac{1}{T} \text { and } \mathcal{S}_{N}(\nu)=\frac{1}{T^{2}} \sum_{n \neq 0} \delta\left(\nu-\frac{n}{T}\right) .
$$

\section{UWB PULSE MODULATIONS}

We now present the shot noise models for the family of UWB pulse modulations and their exact spectrum.

\section{A. $P P M$}

In PPM, the information is carried by the relative positions of the pulses with respect to a regular grid (see for instance [1], [9], [14], [15]). Therefore, a PPM signal can be seen as regularly spaced pulses (regularly spaced point process convoluted with the pulse shape) to which we add random i.i.d. displacement to encode the symbols to be transmitted. The sequence of random times is given by $T_{n}=U+n T, n \in \mathbb{Z}$, where $U$ is a uniform- $[0, T]$ random variable, ensuring the stationarity of the sequence, and $T$ is the time period. The random displacements are modeled as the i.i.d. parameters $\left\{Z_{n}\right\}_{n \in \mathbb{Z}}$. A PPM signal can be written as

$$
X(t)=\sum_{n \in \mathbb{Z}} w\left(t-U-n T-Z_{n}\right)
$$

where $w(t)$ denotes the pulse shape. Hence, we have the shot noise with random excitation in the form of (2), where $A_{n}=1$, $T_{n}=U+n T$, and $h(t, Z)=w(t-Z)$.

In the presence of clock jitter and pulse losses, $X(t)$ becomes

$$
X(t)=\sum_{n \in \mathbb{Z}} Z_{n}^{L} w\left(t-U-n T-Z_{n}^{P}-Z_{n}^{J}\right)
$$

where the sequences of random variables $\left\{Z_{n}^{L}\right\}_{n \in \mathbb{Z}}$ (binary), $\left\{Z_{n}^{P}\right\}_{n \in \mathbb{Z}}$, and $\left\{Z_{n}^{J}\right\}_{n \in \mathbb{Z}}$ model, respectively, the random losses, the position modulation, and the additional displacements due to the clock jitter. As for (8), we have a shot noise with random excitation where now $h(t, Z)=Z^{L} w\left(t-Z^{P}-Z^{J}\right)$, with $Z=\left(Z^{P}, Z^{P}, Z^{J}\right)$. In summary, jitter and losses are easily taken into account through the random filtering function of the shot noise.

Power Spectrum: We have just shown that a PPM signal (8) is a shot noise with random excitation. In particular, $\mathbb{E}\{\widehat{h}(\nu, Z)\}=\phi_{Z}(-\nu) \widehat{w}(\nu)$ and $\mathbb{E}\left\{|\widehat{h}(\nu, Z)|^{2}\right\}=|\widehat{w}(\nu)|^{2}$, where $\phi_{Z}(u)$ is the characteristic function ${ }^{3}$ of the transmitted symbols $\left\{Z_{n}\right\}_{n \in \mathbb{Z}}$. Therefore, from (3) we have

$$
\mathcal{S}_{X}(\nu)=|\widehat{w}(\nu)|^{2}\left[\left|\phi_{Z}(\nu)\right|^{2} \mathcal{S}_{N}(\nu)+\frac{1-\left|\phi_{Z}(\nu)\right|^{2}}{T}\right]
$$

where $\mathcal{S}_{N}$ is the spectrum of a regular $T$ grid, given by (7). For instance, in the case of a transmission of $M$-ary symbols, 
i.i.d. with equal probability, encoded by the relative positions $\{0, T / M, \ldots, T(M-1) / M\}$, we have

$$
\phi_{Z}(\nu)=\frac{1}{M} e^{i \pi \nu T(M-1 / M)} \frac{\sin (\pi \nu T)}{\sin \left(\frac{\pi \nu T}{M}\right)} .
$$

The effect of clock jitter and pulse losses can be straightforwardly taken into account. As an example, consider that the transmitted signal is affected by i.i.d. losses [a special case of (9)]. Then, $\mathbb{E}\{\widehat{h}(\nu, Z)\}=\phi_{Z}(-\nu) \mathbb{E}\left\{Z^{L}\right\} \widehat{w}(\nu)$ and $\mathbb{E}\left\{|\widehat{h}(\nu, Z)|^{2}\right\}=\mathbb{E}\left\{Z^{L^{2}}\right\}|\widehat{w}(\nu)|^{2}$. Therefore, from (3)

$$
\begin{aligned}
\mathcal{S}_{X}(\nu)=|\widehat{w}(\nu)|^{2}\left[\frac{\mathbb{E}\left\{Z^{L}\right\}^{2}\left|\phi_{Z}(\nu)\right|^{2}}{T^{2}} \sum_{n \neq 0} \delta\left(\nu-\frac{n}{T}\right)\right. \\
\left.+\frac{1}{T}\left(\mathbb{E}\left\{Z^{L^{2}}\right\}-\mathbb{E}\left\{Z^{L}\right\}^{2}\left|\phi_{Z}(\nu)\right|^{2}\right)\right] .
\end{aligned}
$$

\section{B. PAM}

A PAM signal transmits the information through the amplitude of the pulses (see for instance [14], [15]). Hence, we have a regularly spaced sequence of pulses with random amplitudes. When the symbols are encoded into a sequence of i.i.d. amplitudes, a PAM signal can be modeled as

$$
X(t)=\sum_{n \in \mathbb{Z}} Z_{n} w(t-U-n T) .
$$

Clearly, (10) is a particular case of a shot noise process in the form of (2), with $A_{n}=1, T_{n}=U+n T$, and $h(t, Z)=Z w(t)$.

If the amplitudes are correlated, we have

$$
X(t)=\sum_{n \in \mathbb{Z}} A_{n} w(t-U-n T)
$$

which is a special case of the shot noise process in (2) with $T_{n}=U+n T$ and $h(t, Z)=w(t)$.

As an example, let us consider a PAM signal with i.i.d. amplitudes affected by random losses of the pulses (the extension to the case of correlated amplitudes is straightforward). In such a case, the PAM signal reads

$$
X(t)=\sum_{n \in \mathbb{Z}} Z_{n}^{A} Z_{n}^{L} w(t-U-n T)
$$

where $Z_{n}^{A}$ are the amplitudes encoding the transmitted symbols while $Z_{n}^{L}$, with values in $\{0,1\}$, are the random losses. Hence, we have a shot noise with random excitation with filtering function $h(t, Z)=Z^{A} Z^{L} w(t)$, where the sequence of i.i.d. parameters is now the random vector $Z_{n}=\left(Z_{n}^{A}, Z_{n}^{L}\right)$.

Power Spectrum: For i.i.d. amplitudes, the PAM model is given by (10). Hence, the sequence of random times forms a regular grid and the filtering function is $h(t, Z)=Z w(t)$, with $\mathbb{E}\{\widehat{h}(\nu, Z)\}=\widehat{w}(\nu) \mathbb{E}\{Z\}$ and $\mathbb{E}\left\{|\widehat{h}(\nu, Z)|^{2}\right\}=$ $|\widehat{w}(\nu)|^{2} \mathbb{E}\left\{|Z|^{2}\right\}$. The power spectrum then reads

$$
\mathcal{S}_{X}(\nu)=|\widehat{w}(\nu)|^{2}|\mathbb{E}\{Z\}|^{2} \mathcal{S}_{N}(\nu)+\frac{1}{T}|\widehat{w}(\nu)|^{2} \operatorname{Var}\{Z\}
$$

where $\mathcal{S}_{N}(\nu)$ is given by (7).
When the amplitudes are correlated (11), by exploiting the fact that a regular grid is a particular case of a renewal process, (4) and (5) give

$$
\mathcal{S}_{X}(\nu)=\frac{|\widehat{w}(\nu)|^{2}}{T^{2}}\left[\mathcal{S}_{A}(T \nu)+|\mathbb{E}\{A\}|^{2} \sum_{n \in \mathbb{Z}} \delta\left(\nu-\frac{n}{T}\right)\right]
$$

where here $\mathcal{S}_{A}$ is the spectral density of the time series. The above formula is also known as the "Bennett-Rice" formula [16].

Here again, various extensions of known results can be obtained by modification of the PAM signal using our modular approach. For instance, from (4) and (5), we can obtain the spectrum of a PAM signal with correlated amplitudes and i.i.d. clock jitter

$$
\begin{aligned}
& \mathcal{S}_{X}(\nu)=\frac{1}{T}|\widehat{w}(\nu)|^{2}\left[1-\left|\phi_{Z}(\nu)\right|^{2}\right] \\
& +\frac{\left|\phi_{Z}(\nu)\right|^{2}|\widehat{w}(\nu)|^{2}}{T^{2}}\left[\mathcal{S}_{A}(T \nu)+|\mathbb{E}\{A\}|^{2} \sum_{n \in \mathbb{Z}} \delta\left(\nu-\frac{n}{T}\right)\right]
\end{aligned}
$$

where $\mathcal{S}_{A}$ is the spectrum of the time series and $\phi_{Z}(u)$ is the characteristic function of the jitter ${ }^{3}$.

\section{PIM}

In PIM, the information is encoded with the relative distance between successive pulses [17]. The sequence of random times $\left\{T_{n}\right\}_{n \in \mathbb{Z}}$ is naturally modeled as a renewal process. A PIM signal reads

$$
X(t)=\sum_{n \in \mathbb{Z}} w\left(t-T_{n}\right)
$$

which is a special case of the shot noise process (2) with $A_{n}=$ $1,\left\{T_{n}\right\}_{n \in \mathbb{Z}}$ being a renewal process, and $h(t, Z)=w(t)$.

Power Spectrum: The spectrum of a PIM signal straightforwardly reads $\mathcal{S}_{X}(\nu)=|\widehat{w}(\nu)|^{2} \mathcal{S}_{N}(\nu)$, where $\mathcal{S}_{N}(\nu)$ is the spectrum (6) of a renewal process (a well know result [12]). For instance, in the case of i.i.d. $M$-ary PIM with equal probabilities, the inter-arrival times take values with equal probabilities over $\{T, 2 T, \ldots, M T\}$, where $T$ is the basic increment of the intervals. Therefore, the characteristic function $\phi_{S}$ and the intensity $\lambda$ appearing in the expression of $\mathcal{S}_{N}(6)$ read

$$
\phi_{S}(\nu)=e^{i \pi \nu T M} \frac{\sin (\pi \nu T(M+1))}{M \sin (\pi \nu T)}, \lambda=\frac{2}{T(M+1)} .
$$

\section{Combination of Pulse Modulations}

Any combination of the basic modulations we have presented can be easily taken into account. In particular, the spectrum of combined modulations can be simply computed from the spectrum of the basic modulations. This remarkable feature is a direct consequence of the modular approach and it is one of the most important aspects of our contribution. We shall present some common situations.

Case 1: PPM/PAM: When the amplitudes are i.i.d. (the correlated case straightforwardly follows), we have

$$
X(t)=\sum_{n \in \mathbb{Z}} Z_{n}^{A} w\left(t-U-n T-Z_{n}^{P}\right)
$$

i.e., a special case of (2) with $A_{n}=1, T_{n}=U+n T, Z=$ $\left(Z^{A}, Z^{P}\right)$, and $h(t, Z)=Z^{A} w\left(t-Z^{P}\right)$. Hence, $\widehat{h}(\nu, Z)=$ $\widehat{w}(\nu) Z^{A} e^{-i 2 \pi \nu Z^{P}}, \mathbb{E}\{\widehat{h}(\nu, Z)\}=\widehat{w}(\nu) \mathbb{E}\left\{Z^{A}\right\} \phi_{Z^{P}}(-\nu)$, 
and $\mathbb{E}\left\{|\widehat{h}(\nu, Z)|^{2}\right\}=|\widehat{w}(\nu)|^{2} \mathbb{E}\left\{\left|Z^{A}\right|^{2}\right\}$, where $\phi_{Z^{P}}(u)$ is the characteristic function $^{3}$ of the i.i.d. displacements $\left\{Z_{n}^{P}\right\}_{n \in \mathbb{Z}}$. The sequence of random times form a regular grid and its spectrum is given by (7). Finally, using (3), the power spectrum of a PPM/PAM signal is given by

$$
\begin{aligned}
\mathcal{S}_{X}(\nu)=|\widehat{w}(\nu)|^{2} & {\left[\frac{\left|\mathbb{E}\left\{Z^{A}\right\}\right|^{2}\left|\phi_{Z^{P}}(\nu)\right|^{2}}{T^{2}} \sum_{n \neq 0} \delta\left(\nu-\frac{n}{T}\right)\right.} \\
+ & \left.\frac{1}{T}\left(\mathbb{E}\left\{\left|Z^{A}\right|^{2}\right\}-\left|\mathbb{E}\left\{Z^{A}\right\}\right|^{2}\left|\phi_{Z^{P}}(\nu)\right|^{2}\right)\right] .
\end{aligned}
$$

Case 2: PIM/PAM: When the amplitudes are i.i.d. (the correlated case straightforwardly follows), we have

$$
X(t)=\sum_{n \in \mathbb{Z}} Z_{n} w\left(t-T_{n}\right)
$$

i.e., a special case of (1) with $A_{n}=1,\left\{T_{n}\right\}_{n \in \mathbb{Z}}$ being a renewal process, and $h(t, Z)=Z w(t)$. We are in the same situation of a PAM signal (10) where the regular grid is replaced by a renewal process. If we consider, as previously done, the i.i.d. $M$-ary PIM/PAM transmission with equal probability, we have $\mathbb{E}\{\widehat{h}(\nu, Z)\}=\widehat{w}(\nu)(M+1) / 2$ and $\mathbb{E}\left\{|\widehat{h}(\nu, Z)|^{2}\right\}=$ $|\widehat{w}(\nu)|^{2} M(M+1)(2 M+1) / 6$. Then, using (3), the power spectrum of a PIM/PAM signal is given by

$$
\begin{aligned}
\mathcal{S}_{X}(\nu)=|\widehat{w}(\nu)|^{2}\left[\left(\frac{M+1}{2}\right)^{2} \mathcal{S}_{N}(\nu)\right. & \\
& \left.+\frac{1}{T}\left(\frac{M(2 M+1)}{3}-\frac{M+1}{2}\right)\right]
\end{aligned}
$$

where the characteristic function $\phi_{S}$ and the intensity $\lambda$ appearing in the expression of $\mathcal{S}_{N}$ (6) are given by (12).

\section{UWB MultiPle-AcCess TeChNIQUeS}

Time hopping and direct sequence are the common multipleaccess techniques employed in UWB systems. We present the shot noise models and the exact power spectrum of pulse modulations combined with such multiple access techniques.

\section{A. Time-Hopping}

General time-hopping (TH) signals are characterized by a deterministic periodic pattern $\left\{c_{n}\right\}$ to allow multiple access. Such a pattern, commonly called signature, determines the positions of the pulses with respect to a regular grid. A general model for TH signals is given by [2]

$$
X(t)=\sum_{n=-\infty}^{\infty} \sum_{l=0}^{L_{c}-1} w\left(t-T_{n}-l T-c_{l} T_{c}\right)
$$

where

- $\left\{c_{l}\right\}, l=0, \ldots, L_{c}$, is the signature sequence characterizing the $L_{c}$-periodic pattern;

- $T$ is the spacing of the regular grid $\left(T_{c}<T\right)$;

- $T_{n}$ is a sequence of random times that depends on the type of temporal modulation (usually PPM).
Equation (13) is a special case of a shot noise process with random times $T_{n}$, and filtering function $h(t, Z)=$ $\sum_{l=0}^{L_{c}-1} w\left(t-l T-c_{l} T_{c}\right){ }^{4}$ We consider two cases.

Case 1: Time-Hopping With PPM/PAM: Let $\left\{Z_{n}^{P}\right\}_{n \in \mathbb{Z}}$ denote a sequence of i.i.d. random variables representing the positions in PPM, and $\left\{Z_{n}^{A}\right\}_{n \in \mathbb{Z}}$ denote a sequence of i.i.d. random variables representing the amplitudes in PAM (for simplicity we consider the case of i.i.d. amplitudes; the correlated case can be easily extended). The random times $T_{n}$ form a regular grid

$$
T_{n}=U+n L_{c} T
$$

where $U$ is a random variable uniformly distributed over $\left[0, L_{c} T\right]$. A time-hopping signal using PPM/PAM can be then written as [2]

$$
X(t)=\sum_{k=-\infty}^{\infty} Z_{n}^{A} \sum_{l=0}^{L_{c}-1} w\left(t-T_{n}-Z_{n}^{P}-l T-c_{l} T_{c}\right)
$$

with $T_{n}$ as in (14). Again, we have a shot noise with random excitation (2), with random times given by (14), $A_{n}=1, Z=$ $\left(Z^{A}, Z^{P}\right)$, and

$$
h(t, Z)=Z^{A} \sum_{l=0}^{L_{c}-1} w\left(t-Z^{P}-l T-c_{l} T_{c}\right) .
$$

Case 2: Time-Hopping PPM/PAM Signal With Jitter and Thinning: As previously seen for PPM and PAM, i.i.d. jitter and thinning are introduced using the random parameter of the impulse response (we remark that the case of correlated losses can be modeled through the correlated sequence $\left\{A_{n}\right\}_{n \in \mathbb{Z}}$, with values in $\{0,1\}$ ). More precisely, we introduce the i.i.d. sequences of i.i.d. $L_{c}$-vectors

$$
\left\{\left(Z_{0 ; n}^{J}, \ldots, Z_{L_{c}-1 ; n}^{J}\right)\right\} \text { and }\left\{\left(Z_{0 ; n}^{L}, \ldots, Z_{L_{c}-1 ; n}^{L}\right)\right\}
$$

to model, respectively, the random displacements and the random losses ( $Z^{L}$ is a binary sequence). We remark that the introduction of $L_{c}$ vectors is necessary in order to consider jitter and thinning of each pulse of the signature. ${ }^{5}$

Then, a time-hopping PPM/ PAM signal reads

$$
\begin{aligned}
X(t)=\sum_{n \in \mathbb{Z}} Z_{n}^{A} & \sum_{l=0}^{L_{c}-1} Z_{l ; n}^{L} \\
& \times w\left(t-T_{n}-Z_{n}^{P}-l T-c_{l} T_{c}-Z_{l ; n}^{J}\right)
\end{aligned}
$$

with $T_{n}$ as in (14). Again, it is a shot noise with random excitation, where the sequence of random parameters is

$$
Z_{n}=\left(Z_{n}^{A}, Z_{n}^{P},\left(Z_{0 ; n}^{J}, \ldots, Z_{L_{c}-1 ; n}^{J}\right),\left(Z_{0 ; n}^{L}, \ldots, Z_{L_{c}-1 ; n}^{L}\right)\right)
$$

the filtering function is

$$
h(t, Z)=Z^{A} \sum_{l=0}^{L_{c}-1} Z_{l}^{L} w\left(t-Z^{P}-Z_{l}^{J}-l T-c_{l} T_{c}\right)
$$

and the random times are given by (14). Note that expression (17) is an extension of the model presented in [2] that easily takes into account random losses of the pulses, as well as generic jitter.

${ }^{4}$ Alternatively, the underlying sequence of random times can be seen as $T_{n}+$ $\sum_{l=0}^{L_{c}-1}\left(l T+c_{l} T_{c}\right)$, i.e., a cluster point process [18] with seeds $T_{n}$ and deterministic clusters $\sum_{l=0}^{L_{c}-1}\left(l T+c_{l} T_{c}\right)$.

${ }^{5}$ This is due to the clustered structure of a TH sequence. 
Power Spectrum: We consider the time-hopping PPM/PAM signal with jitter and losses of (17) (Case 2). Note that the signal without jitter and losses (Case 1) can be treated as a special case. The spectrum is straightforwardly obtained from (3) by recalling that the underlying point process is now a regular $L_{c} T$-grid and that the random filtering function is given by (18). Therefore, $\mathcal{S}_{N}$ is given by (6), where $T$ is replaced by $L_{c} T$, and first and second order moments of $\widehat{h}(\nu, Z)$ are given by (19), as shown at the bottom of the page. The power spectrum is then given by (20), as shown at the bottom of the page.

\section{B. Direct-Sequence}

Like the time-hopping, a direct-sequence signal can be used for multiple access. It consists of multiplying a stream of pulses by a periodic deterministic sequence of +1 and -1 (the signature). It can be generally modeled as

$$
X(t)=\sum_{n \in \mathbb{Z}} \sum_{k=0}^{L_{d}-1} a_{k}^{d s} w\left(t-T_{n L_{d}+k}\right)
$$

where

- $L_{d}$ is the period of the signature sequence;

- $a_{k}^{d s}, k=0, \ldots, L_{d}-1$, is the signature sequence, where $a^{d s} \in\{+1,-1\}$.

Next, we consider the TH/DS with PPM/ PAM in the presence of jitter and random losses. The corresponding model can be obtained by adding the DS feature to the TH signal with PPM/ PAM of the previous section. By assuming that the period $L_{c}$ of the TH signature and the period $L_{d}$ of the DS signature are multiple of each other, it is possible to easily consider the cases $L_{c}>L_{d}$ and $L_{c}<L_{d}$ (see [10]). For simplicity, but without loss of generality, we consider the situation where $L_{c}=L_{d}$ and we call $L$ such common period. As for the TH signal of Section IV-A, we introduce the i.i.d. sequences of i.i.d. L-vectors

$$
\left\{\left(Z_{0 ; n}^{J}, \ldots, Z_{L-1 ; n}^{J}\right)\right\} \text { and }\left\{\left(Z_{0 ; n}^{L}, \ldots, Z_{L-1 ; n}^{L}\right)\right\}
$$

to model, respectively, the random jitter and the random losses. Let $\left\{Z_{n}^{P}\right\}_{n \in \mathbb{Z}}$ denote the i.i.d. sequence modeling the positions of PPM and let $\left\{Z_{n}^{A}\right\}_{n \in \mathbb{Z}}$ denote the i.i.d. sequence modeling the amplitudes of PAM.
Then, a TH/DS signal with PPM/PAM is given by (22)

$$
\begin{aligned}
& X(t)=\sum_{n \in \mathbb{Z}} Z_{n}^{A} \sum_{k=0}^{L-1} Z_{k ; n}^{L} a_{k}^{d s} \\
& \quad \times w\left(t-U-n L T-Z_{n}^{P}-k T-c_{k} T_{c}-Z_{k ; n}^{J}\right) .
\end{aligned}
$$

i.e., it a shot noise with random excitation (2) with $A_{n}=1$, $Z=\left(Z^{A}, Z^{P},\left(Z_{0}^{J}, \ldots, Z_{L-1}^{J}\right),\left(Z_{0}^{L}, \ldots, Z_{L-1}^{L}\right)\right)$, and

$$
h(t, Z)=Z^{A} \sum_{k=0}^{L-1} Z_{k}^{L} a_{k}^{d s} w\left(t-Z^{P}-k T-c_{k} T_{c}-Z_{k}^{J}\right)
$$

Power Spectrum: We consider the shot noise model (22) of the TH/DS signal with PPM/PAM in the presence of jitter and random losses. Here again, the power spectrum can be straightforwardly obtained from (3). The underlying point process is a regular $L T$-grid, therefore, $\mathcal{S}_{N}$ is given by (6), where $T$ is replaced by $L T$. The first and second order moments of the random filtering function (23) are given by (24).

$$
\begin{gathered}
\mathbb{E}\{\widehat{h}(\nu, Z)\}=\sum_{k=0}^{L-1} a_{k}^{d s} e^{-i 2 \pi \nu\left(l T+c_{l} T_{c}\right)} \\
\times \widehat{w}(\nu) \mathbb{E}\left\{Z^{A}\right\} \mathbb{E}\left\{Z^{L}\right\} \phi_{Z^{P}}(-\nu) \phi_{Z^{J}}(-\nu) \\
\mathbb{E}\left\{|\widehat{h}(\nu, Z)|^{2}\right\}=|\widehat{w}(\nu)|^{2} \mathbb{E}\left\{\left|Z^{A}\right|^{2}\right\} \\
\times\left(L \mathbb{E}\left\{\left|Z^{L}\right|^{2}\right\}+\left|\mathbb{E}\left\{Z^{L}\right\}\right|^{2}\left|\phi_{Z^{P}}(\nu)\right|^{2}\right. \\
\left.\times\left(\left|\sum_{l=0}^{L-1} a_{l}^{d s} e^{-i 2 \pi \nu\left(l T+c_{l} T_{c}\right)}\right|^{2}-L\right)\right) .
\end{gathered}
$$

Finally, the power spectral density of a TH/DS with PPM/ PAM in the presence of jitter and random losses (22) is given by (25), as shown at the bottom of the next page. We remark that the parameters characterizing the different features of the model (signature of DS and TH, type of modulations, as well as the characteristic of the jitter and the random losses) appear clearly and separately.

Spectrum of TH/DS with PPM/PAM was not previously available in the literature. We emphasize the generality of the

$$
\begin{aligned}
\mathbb{E}\{\widehat{h}(\nu, Z)\}= & L_{c} \widehat{w}(\nu) \mathbb{E}\left\{Z^{A}\right\} \mathbb{E}\left\{Z^{L}\right\} \phi_{Z^{P}}(-\nu) \phi_{Z^{J}}(-\nu) \sum_{l=0}^{L_{c}-1} e^{-i 2 \pi \nu\left(l T+c_{l} T_{c}\right)} \\
\mathbb{E}\left\{\left.\widehat{h}(\nu, Z)\right|^{2}\right\}= & |\widehat{w}(\nu)|^{2} \mathbb{E}\left\{\left|Z^{A}\right|^{2}\right\}\left[L_{c} \mathbb{E}\left\{\left|Z^{L}\right|^{2}\right\}+\left|\mathbb{E}\left\{Z^{L}\right\}\right|^{2}\left|\phi_{Z^{J}}(\nu)\right|^{2}\left(\left|\sum_{l=0}^{L_{c}-1} e^{-i 2 \pi \nu\left(l T+c_{l} T_{c}\right)}\right|^{2}-L_{c}\right)\right] \\
\mathcal{S}_{X}(\nu)=|\widehat{w}(\nu)|^{2} \frac{1}{L_{c} T} & \\
\times & {\left[\left|\mathbb{E}\left\{Z^{A}\right\}\right|^{2}\left|\mathbb{E}\left\{Z^{L}\right\}\right|^{2}\left|\phi_{Z^{P}}(\nu)\right|^{2}\left|\phi_{Z^{J}}(\nu)\right|^{2}\left|\sum_{l=0}^{L_{c}-1} e^{-i 2 \pi \nu\left(l T+c_{l} T_{c}\right)}\right|^{2} \frac{1}{L_{c} T} \sum_{n \neq 0} \delta\left(\nu-\frac{n}{L_{c} T}\right)\right.} \\
& +L_{c} \mathbb{E}\left\{\left|Z^{A}\right|^{2}\right\}\left(\mathbb{E}\left\{\left|Z^{L}\right|^{2}\right\}-\left|\mathbb{E}\left\{Z^{L}\right\}\right|^{2}\left|\phi_{Z^{J}}(\nu)\right|^{2}\right) \\
& \left.+\left|\mathbb{E}\left\{Z^{L}\right\}\right|^{2}\left|\phi_{Z^{J}}(\nu)\right|^{2}\left|\sum_{l=0}^{L_{c}-1} e^{-i 2 \pi \nu\left(l T+c_{l} T_{c}\right)}\right|^{2}\left(\mathbb{E}\left\{\left|Z^{A}\right|^{2}\right\}-\left|\mathbb{E}\left\{Z^{A}\right\}\right|^{2}\left|\phi_{Z^{P}}(\nu)\right|^{2}\right)\right]
\end{aligned}
$$


result that presents generic $\mathrm{TH}$ and DS, generic jitter, and generic losses. Moreover, different pulse modulations can be taken into account simply by replacing the spectrum $\mathcal{S}_{N}$ and the random pulse function.

\section{NUMERICAL RESULTS}

The modularity of the spectral formula has allowed us to easily write a generic Matlab toolbox ${ }^{6}$ for the numerical evaluation of the spectrum of UWB signals, including the case where the signal is transmitted over a multipath fading channel. The toolbox enables us to specify different pulse modulations and different phenomena affecting the transmission. We present the numerical evaluation of the power spectrum of a PPM signal with $\mathrm{TH}$ and DS, in the presence of jitter and random losses. The characteristics of the signal are the following:

- Gaussian derivative pulse shape with width $\equiv 0.3 \mathrm{~ns}$;

- $\quad$ chip-time $T_{\mathrm{CHIP}}=6 \mathrm{~ns}$;

- binary symbols coded into delays of $0 \mathrm{~ns}$ and $12 \mathrm{~ns}$;

- $\quad$ TH signature with pulses at [1 2568111215$] \times T_{\mathrm{CHIP}}$;

- $\quad$ DS signature $=\left[\begin{array}{llllllll}1 & -1 & 1 & 1 & 1 & -1 & 1 & -1\end{array}\right]$ with pulses at

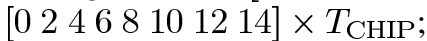

- jitter of $[-10,-8,-6,-4,-2,0,2,4,6,8,10] \times 0.001 \mathrm{~ns}$ with equal probability;

- losses with probability 0.1 .

Fig. 1 depicts the power spectrum of (a) PPM signal, (b) PPM signal with jitter and losses, (c) TH- PPM signal with jitter and losses, and (d) DS- PPM signal with jitter and losses. We note

\footnotetext{
${ }^{6}$ The code can be freely downloaded (under the GNU GPL license) at http://lcavwww.epfl.ch/software.
}

the presence of spectral lines due to the underlying periodicity of the random times, and therefore due to the term $S_{N}$. From (20) and (25), or more generally from (3), we remark that in the presence of an i.i.d. PAM, the term $S_{N}$ is multiplied by the average value of the amplitudes. By using a BPSK modulation $(\{-1,1\}$ PAM with equal probability), such an average value is zero and consequently the term containing the spectral lines is null.

\section{CONCLUSION}

Shot noise processes aptly model the large family of UWB signals, and associated spectrum expressions provide a general formula for evaluating the spectra of UWB transmissions. The spectral formulas we have presented unify well-known results and provide spectral expressions for highly complicated UWB signals. Moreover, the corresponding approach is modular, allowing us to progressively add specific features to the model in a systematic manner. In addition, the exact power spectrum expressions we have derived are easy to understand as the contribution of the various features of the model appear explicitly and separately. Such a feature of our approach is useful in the design and analysis of UWB systems.

\section{APPENDIX}

Proof: (Theorem II.1) Let $X(t)$ be the shot noise of (1) and let $Z$ be a random variable with distribution equal to the the common distribution of the i.i.d. random variables $\left\{Z_{n}\right\}_{n \in \mathbb{Z}}$. Call $\mathbb{E}_{Z}$ the expectation with respect to such a distribution. Then, (see equation at the bottom of the page).

$$
\begin{aligned}
\mathcal{S}_{X}(\nu)=|\widehat{w}(\nu)|^{2} \frac{1}{L T} & \\
& \times\left(\left|\mathbb{E}\left\{Z^{A}\right\}\right|^{2}\left|\mathbb{E}\left\{Z^{L}\right\}\right|^{2}\left|\phi_{Z^{P}}(\nu)\right|^{2}\left|\phi_{Z^{J}}(\nu)\right|^{2}\left|\sum_{l=0}^{L-1} a_{l}^{d s} e^{-i 2 \pi \nu\left(l T+c_{l} T_{c}\right)}\right|^{2} \frac{1}{L T} \sum_{n \neq 0} \delta\left(\nu-\frac{n}{L T}\right)\right. \\
& +L \mathbb{E}\left\{\left|Z^{A}\right|^{2}\right\}\left(\mathbb{E}\left\{\left|Z^{L}\right|^{2}\right\}-\left|\mathbb{E}\left\{Z^{L}\right\}\right|^{2}\left|\phi_{Z^{J}}(\nu)\right|^{2}\right)+\left|\mathbb{E}\left\{Z^{L}\right\}\right|^{2}\left|\phi_{Z^{J}}(\nu)\right|^{2}\left|\sum_{l=0}^{L-1} a_{l}^{d s} e^{-i 2 \pi \nu\left(l T+c_{l} T_{c}\right)}\right|^{2} \\
& \left.\times\left(\mathbb{E}\left\{\left|Z^{A}\right|^{2}\right\}-\left|\mathbb{E}\left\{Z^{A}\right\}\right|^{2}\left|\phi_{Z^{P}}(\nu)\right|^{2}\right)\right)
\end{aligned}
$$

$$
\begin{aligned}
\operatorname{cov}(X(t+\tau), X(t))= & \mathbb{E}\left\{\sum_{n \in \mathbb{Z}} \mathbb{E}_{Z}\left\{h\left(t+\tau-T_{n}, Z\right) h^{*}\left(t-T_{n}, Z\right)\right\}\right\} \\
+ & \mathbb{E}\left\{\sum_{n \in \mathbb{Z}} \sum_{k \in \mathbb{Z}} \mathbb{E}_{Z}\left\{h\left(t+\tau-T_{n}, Z\right)\right\} \mathbb{E}_{Z}\left\{h^{*}\left(t-T_{k}, Z\right)\right\}\right\} \\
& -\mathbb{E}\left\{\sum_{n \in \mathbb{Z}} \mathbb{E}_{Z}\left\{h\left(t+\tau-T_{n}, Z\right)\right\} \mathbb{E}_{Z}\left\{h^{*}\left(t-T_{n}, Z\right)\right\}\right\} \\
& -\mathbb{E}\left\{\sum_{n \in \mathbb{Z}} \mathbb{E}_{Z}\left\{h\left(t+\tau-T_{n}, Z_{n}\right)\right\}\right\} \mathbb{E}\left\{\sum_{k \in \mathbb{Z}} \mathbb{E}_{Z}\left\{h^{*}\left(t-T_{k}, Z_{k}\right)\right\}\right\} \\
= & A+B-C-D .
\end{aligned}
$$



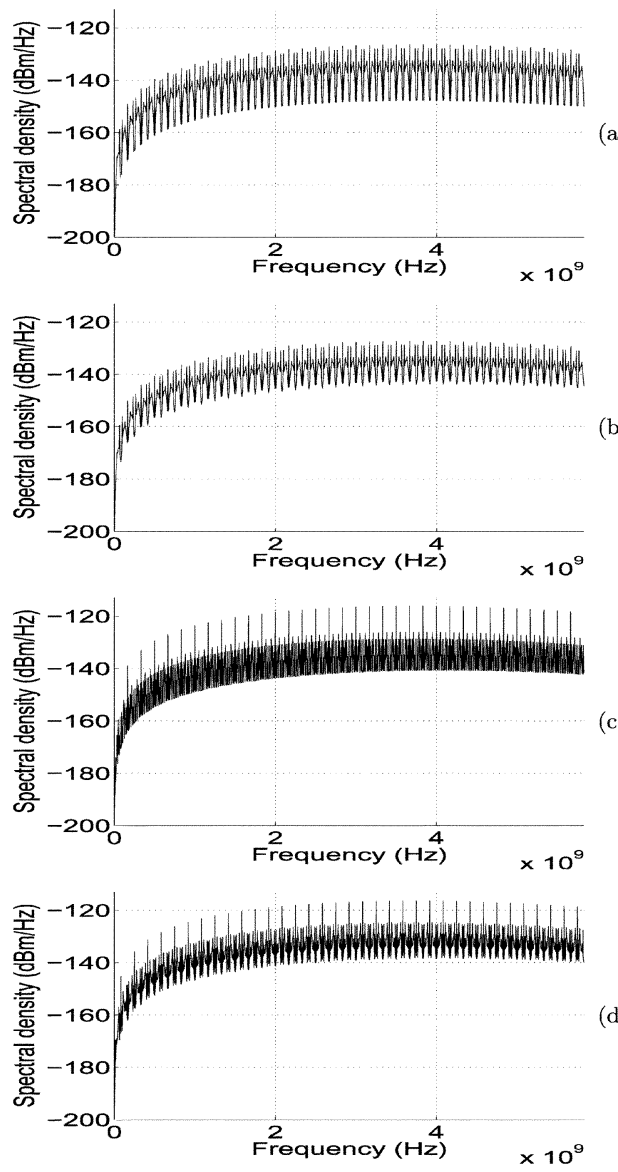

Fig. 1. Spectrum of (a) PPM signal, (b) PPM signal with jitter and losses, (c) TH-PMM signal with jitter and losses, and (d) DS-PPM signal with jitter and losses.

\section{By Campbell's theorem [12]}

$$
\begin{aligned}
& A=\lambda \int_{\mathbb{R}} \mathbb{E}\left\{h(t+\tau-s, Z) h^{*}(t-s, Z)\right\} d s \\
& C=\lambda \int_{\mathbb{R}} \mathbb{E}\{h(t+\tau-s, Z)\} \mathbb{E}\left\{h^{*}(t-s, Z)\right\} d s,
\end{aligned}
$$

and by Parseval's equality

$$
\begin{aligned}
& A=\lambda \int_{\mathbb{R}} e^{i 2 \pi \nu \tau} \mathbb{E}\left\{\widehat{h}(\nu, Z) \widehat{h}^{*}(\nu, Z)\right\} d \nu \\
& C=\lambda \int_{\mathbb{R}} e^{i 2 \pi \nu \tau} \mathbb{E}\{\widehat{h}(\nu, Z)\} \mathbb{E}\left\{\widehat{h}^{*}(\nu, Z)\right\} d \nu .
\end{aligned}
$$

Therefore, $A-C=\lambda \int_{\mathbb{R}} e^{i 2 \pi \nu \tau} \operatorname{Var}\{\widehat{h}(\nu, Z)\} d \nu$. By the definition of the Bartlett spectral density $\mathcal{S}_{N}$ [12]

$$
B-D=\int_{\mathbb{R}} e^{i 2 \pi \nu \tau}|\mathbb{E}\{\widehat{h}(\nu, Z)\}|^{2} \mathcal{S}_{N}(\nu) d \nu .
$$

Finally

$$
\begin{aligned}
\operatorname{cov}(X(t+\tau), X(t)) & =\lambda \int_{\mathbb{R}} e^{i 2 \pi \nu \tau} \operatorname{Var}\{\widehat{h}(\nu, Z)\} d \nu \\
& +\int_{\mathbb{R}} e^{i 2 \pi \nu \tau}|\mathbb{E}\{\widehat{h}(\nu, Z)\}|^{2} \mathcal{S}_{N}(\nu) d \nu
\end{aligned}
$$

and by the definition of the power spectrum of a WSS process [19], [20] we obtain

$$
\mathcal{S}_{X}(\nu)=|\mathbb{E}\{\widehat{h}(\nu, Z)\}|^{2} \mathcal{S}_{N}(\nu)+\lambda \operatorname{Var}\{\widehat{h}(\nu, Z)\}
$$

i.e., (3). Similarly, we obtain the proof of (4), where the Bartlett spectrum is replace by the spectrum of a modulated renewal process (see [10] for more details).

\section{ACKNOWLEDGMENT}

The authors wish to thank M. Vetterli, P. Brémaud, W. Suwansantisuk, P. Pinto, J. Salez, and I. Keliher for their helpful suggestions and careful reading of the manuscript.

\section{REFERENCES}

[1] M. Win and R. Scholtz, "Impulse radio: How it works," IEEE Commun. Lett., vol. 2, no. 2, pp. 36-38, Feb. 1998.

[2] M. Win, "A unified spectral analysis of generalized time-hopping spread-spectrum signals in the presence of timing jitter,' IEEE J. Sel. Areas Commun., vol. 20, no. 9, pp. 1664-1676, Dec. 2002.

[3] J. Romme and L. Piazzo, "On the power spectral density of time-hopping impulse radio," in IEEE Conf. Ultra Wide-band Systems Technologies, May 21-23, 2002, pp. 241-244.

[4] G. Cariolaro, T. Erseghe, and L. Vangelista, "Exact spectral evaluation of the family of digital pulse interval modulated signals," IEEE Trans. Inf. Theory, vol. 47, no. 11, pp. 2983-2992, Nov. 2001.

[5] L. Bondesson, "shot noise processes and shot noise distributions," in Encyclopedia of Statistical Sciences, N. L. J. S. Kotz, Ed. New York: Wiley, 1988, vol. 8, pp. 448-452.

[6] M. Parulekar and A. M. Makowski, " $M / G / \infty$ input processes: A versatile class of models for network traffic," in Proc. INFOCOM, vol. 2, Apr. 7-11, 1997, pp. 419-426.

[7] D. Snyder and C. Georghiades, "Design of coding and modulation for power-efficient use of a band-limited optical channel," IEEE Trans. Commun., vol. 31, no. 4, pp. 560-565, Apr. 1980.

[8] C. Georghiades, "Some optimal and suboptimal receivers deriving information from nonsynchronized poisson data," IEEE Trans. Inf. Theory, vol. 35 , no. 1 , pp. 157-165, Jan. 1989.

[9] M. Win and R. Scholtz, "Ultra-wide bandwidth time-hopping spreadspectrum impulse radio for wireless multiple-access communications," IEEE Trans. Commun., vol. 48, no. 4, pp. 679-689, Apr. 2000.

[10] A. Ridolfi, "Power spectra of random spikes and related complex signals, with application to communications," Ph.D. dissertation, Ecole Polytechnique Fédérale de Lausanne, Lausanne, Switzerland, 2004.

[11] A. Ridolfi and M. Z. Win, "Power spectra of multipath faded pulse trains," in Proc. IEEE Int. Symp. Inf. Theory, Sep. 4-9, 2005, pp. 102-106.

[12] D. J. Daley and D. Vere-Jones, An Introduction to the Theory of Point Processes, 2nd ed. New York: Springer-Verlag, 2002, vol. I.

[13] S. I. Resnick, Adventures in Stochastic Processes. Boston, MA: Birkhauser, 1994.

[14] D. Middleton, Statistical Communication Theory. New York: McGraw-Hill, 1960.

[15] M. Schwartz, W. R. Bennet, and S. Stein, Communication Systems and Techniques. New York: McGraw-Hill, 1966.

[16] W. R. Bennett and S. O. Rice, "Spectral density and autocorrelation functions associated with binary frequency-shift keying," Bell Syst. Tech. J., vol. 42, pp. 2355-2385, 1963.

[17] Z. Ghassemlooy, A. R. Hayes, N. L. Seed, and E. D. Kaluarachchi, "Digital pulse interval modulation for optical communications," IEEE Commun. Mag., vol. 35, no. 12, pp. 95-99, Dec. 1998.

[18] D. Cox and V. Isham, Point Processes: Chapman \& Hall, 1980.

[19] S. Bochner, Harmonic Analysis and the Theory of Probability. Berkeley: Univ. California Press, 1955.

[20] D. Dacunha-Castelle and M. Duflo, Probability and Statistics. New York: Springer-Verlag, 1986, vol. 2.

Andrea Ridolfi (S'04) photograph and biography not available at the time of publication.

Moe Z. Win (S'85-M'87-SM'97-F'04) photograph and biography not available at the time of publication. 